\title{
Benign Fibrous Histiocytoma of Mandible: A Pathogenetic \& Diagnostic Perspective
}

\author{
Seema Salve ${ }^{1}$, Jyoti Bhavthankar ${ }^{2}$, Mandakini Mandale \\ ${ }^{1}$ (Government Dental College \& Hospital, Aurangabad, Maharashtra, India) \\ ${ }_{2}^{2}$ (Government Dental College \& Hospital, Aurangabad, Maharashtra, India)
}

\begin{abstract}
Fibrous histiocytoma is a heterogenous group of mesenchymal tumors that is primarily composed of fibroblasts \& histiocytes. The most common site of occurrence of these lesions is on the skin of extremities. Intraoral benign fibrous histiocytomas (BFH)s are commonly seen in the soft tissues of the buccal mucosa, gingiva, lips, soft palate \& floor of the mouth. However its occurrence in deeper soft tissues (less than 1\%) \& intrabony locations is not unusual. Jaw bones are found to be the rarest sites, as till date only two cases in maxilla \& six in mandible have been reported. Also BFH represents a pathologist's phantasm because histologically \& morphologically it mimics many other soft tissue tumors. Because of lack of presence of specific immunohistochemical marker for BFH, diagnosis is confirmed on exclusion basis. A case of BFH at an unusual location - mandibular paraosteum is reported here along with its pathogenetic \& diagnostic perspective.
\end{abstract}

Keywords: Benign Fibrous Histiocytoma, fibroblasts, histiocytes

\section{Introduction}

Fibrous histiocytoma is a mesenchymal tumor composed of a mixture of fibroblastic \& histiocytic cells that are often arranged in a cartwheel or storiform pattern, accompanied by a varying number of inflammatory cells, foam cells \& siderophages. ${ }^{[1]}$ This tumor mostly occurs in the dermis \& superficial cutis but also found in deep soft tissues \& occasionally in parenchymal organs. ${ }^{[1]}$ It was first described by Stout \& Lattes in 1967. ${ }^{[2]}$ Because of variable nature of this lesion, an array of terms has been used for it including dermatofibroma, histiocytoma cutis, nodular superficial fibrosis, sclerosing hemangioma, xanthogranuloma \& fibroxanthoma. ${ }^{[3,4]}$ A malignant counterpart of this tumor 'Malignant $\mathrm{FH}$ ' is also known as 'Pleomorphic sarcoma' ${ }^{[5]}$

Depending on its location this tumor is classified into two types: fibrohistiocytic tumors of soft tissues which are further subdivided into cutaneous \& noncutaneous types and secondly as fibrohistiocytic tumors of the bone. ${ }^{[6]}$

Most common sites of this tumor comprise lower extremities (50\%), upper extremities (20\%), retroperitoneum $(20 \%)$, with rare occurrence in pelvis, knee and head \& neck. ${ }^{[6]}$ Oral \& perioral lesions are uncommon. In the oral cavity the common sites include buccal mucosa, gingiva, lips, soft palate \& floor of the mouth. Intrabony lesions within the jaws are extremely rare. Till date only two cases of maxilla \& six cases of mandible have been reported. ${ }^{[2]}$ No case has been reported in a paraosteal location. It usually occurs in middle aged women (under 50 years of age). Herewith reported is a case of BFH in the mandibular paraosteum in a 12 year old male child along with its pathogenetic, diagnostic \& therapeutic approach.

\section{Case Report}

A 12 year old male child reported with a chief complaint of mild pain \& slow growing swelling on the right side of face near the lower jaw since one year. The pain was of dull \& intermittent type. Past medical, psychosocial \& family history of the patient were insignificant. Extraoral examination revealed a well defined oval swelling of size $4 \mathrm{~cm} \mathrm{X} 3 \mathrm{~cm}$ over the buccal surface of mandible in the parasymphysis region on right side (Fig. $1 \mathrm{~A}, \mathrm{~B})$. The swelling was hard in consistency with smooth surface texture. The surface temperature was normal. Regional lymph nodes were non palpable. Intraoral examination revealed mixed dentition with good oral hygiene. Teeth \& oral mucosa were found to be absolutely normal \& intact (Fig. 1 C). A cone beam computed tomography revealed an expansile lesion of $23 \times 13 \times 18 \mathrm{~mm}$ size with sclerotic border on mandibular buccal surface in relation to $84,85 \& 46$. Buccal expansion was evident \& erosion was seen at superior aspect. The $3 \mathrm{D}$ image of CBCT revealed intrabony destruction in relation to $84,85 \& 46$. Lamina dura of the teeth was intact (Fig. 1 D).

Pulp vitality testing for $84,85 \& 46$ showed normal response.

Thus based on clinical \& radiographic examination a provisional diagnosis of nonossifying fibroma or benign odontogenic tumor was considered. 
The lesion was excised surgically under local anaesthesia. The lesion was removed with 2 mm free margins by reflecting the flap. Primary closure of the wound was established using 3-0 mersilk. Elastic pressure bandage was given for support extraorally. Antibiotic coverage \& chlorhexidine gluconate mouthwash were prescribed for prophylactic purpose. The postoperative course was uneventful.

The specimen consisted of an oval encapsulated mass measuring $4 \mathrm{~cm} \times 2.5 \mathrm{~cm}$ (Fig. $2 \mathrm{~A}$ ). Grossly it showed yellow- brown mass which was soft to firm in consistency \& with rough surface texture. The cut surface revealed white to yellow smooth shiny surface (Fig. 2 B).

Microscopically the lesion was chiefly composed of fibroblast like spindle cells having tapered \& blunt ends with plump \& vesiculated nucleus. The cells were arranged mainly in interlacing fascicles or in a storiform pattern at focal places (Fig. 2C). Plump, round to polygonal histiocytic cells were found intervening the spindle cells (Fig. 2D). Touton type multinucleated giant cells (Fig. 2 E), mild to moderate degree of vascularity \& chronic inflammatory cell infiltrate composed of lymphocytes \& plasma cells were evident. There was no evidence of any increased or abnormal mitotic activity, cellular pleomorphism, nuclear atypia or necrosis. As the histologic picture did not reveal any odontogenic islands, the clinical diagnosis of odontogenic tumor was ruled out \& the lesion was diagnosed as cellular variant of Benign Fibrous Histiocytoma.

Since BFH shares histopathologic features with many other lesions like solitary fibrous tumor, nodular fasciitis, neurofibroma, leiomyoma, malignant fibrous histiocytoma, fibrosarcoma, leiomyosarcoma etc, the diagnosis was confirmed by IHC. As there is no specific marker for BFH. Hence its diagnosis is based on exclusion.

Considering the unusual site of the tumor in this case, immunohistochemistry was performed to confirm the diagnosis. It revealed positivity for SMA, CD 68, less than $10 \%$ positivity for Ki 67 \& negativity for S 100 (Fig. 2 F, G, H, I ). This indicated fibroblastic \& histiocytic cell origin, less aggressive behavior \& excluded the neural lesion \& thus confirmed the BFH.

One year follow up revealed no signs of local recurrence.

\section{Discussion}

The common age of occurrence for $\mathrm{BFH}$ is usually in the fifth decade with male to female ratio of 2.5:1. The etiology of oral BFH is obscure. Chronic irritation, continuous trauma \& spontaneous development have been suggested as the possible etiological factors. ${ }^{[8]}$

The precise mechanism for the development of BFH is unkown. Cell of origin as evidenced by tissue culture is histiocytes which act as facultative fibroblasts.

The cell surface proteoglycan, syndecan $1 \&$ fibroblast growth factor receptor 2 involved in epithelial mesenchymal cross stalk, may play a role in the growth of BFH. Transforming growth factor $\beta$ signaling might be a trigger of fibrosis seen in BFH. TGF $\beta$ along with other fibrinogenic factors may be produced by mast cells which have been reported to occur in abnormally high numbers in BFH. ${ }^{[7]}$ Study by Plaszczyca A stated that $\mathrm{BFH}$ tumorigenesis may be due to distorted protein kinase $\mathrm{C}$ activity. ${ }^{[7]} \mathrm{BFHs}$ were found to harbor recurrent fusions of genes encoding membrane-associated proteins (podoplanin, CD63 and LAMTOR1) with genes encoding protein kinase $\mathrm{C}$ (PKC) isoforms PRKCB and PRKCD.

Light microscopy of the lesion reveal dual cell population of fibroblasts \& histiocytes. Electron microscopic \& ultrastructural studies also demonstrated two cell populations i.e. one resembling fibroblasts with organized lamellae of rough endoplasmic reticulum, few or no lipid droplets \& no phagolysosomes. ${ }^{[1]}$ These studies also demonstrated that the histiocytic \& fibroblastic cell lines were derived from the small number of the undifferentiated mesenchymal cells.

Apart from this immunohistochemical studies with HSP47, CD 68 \& factor XIIIa supported the fibroblastic, histiocytic \& dermal dendritic origins. ${ }^{[7]}$

These tumors usually present as slow growing, solitary nodule on the suface of extremities. The oral lesions are typically found in the middle aged $\&$ older adults $\&$ vary in size from few mms to several cms. Deeper tumors tend to be larger. They are usually asymptomatic but itching or pain often are noted. ${ }^{[3]}$

Histology usually depicts the benign nature of fibrous histiocytoma. The lesion characteristically composed of predominant fibroblasts \& histiocytes with sporadical multinucleated giant cells, lipid containing xanthoma cells \& lymphocytes. Spindle shaped fibroblasts are usually arranged in fascicular or in storiform pattern. Cellular \& nuclear pleomorphism \& mitosis are extremely rare. The occasional pleomorphic cells with hyperchromatic nuclei and clear to eosinophilic cytoplasm referred to by some as "monster cells," seem to be a degenerative phenomenon and do not affect the prognosis adversely. ${ }^{[1]}$ The connective tissue stroma is usually densely fibrous \& may show myxoid or hyalinized changes at focal places. ${ }^{[1]}$ However, the presence of both pleomorphism and mitotic activity suggest malignancy. ${ }^{[1]}$

Histological variants of BFH include cellular, epitheloid \& aneurysmal as three main variants along with clear cell, lipidized, palisading, myxoid \& granular cell types. ${ }^{[1]}$ In addition Han et al classified it as fibrocollagenous, histiocytic, cellular, aneurysmal, angiomatous, sclerotic, monster, palisading \& keloidal 
types. ${ }^{[9]}$ Amongst all these cellular variant is found to have most aggressive behavior \& high risk of local recurrence $(25 \%) \&$ often poses a diagnostic challenge to distinguish it from more aggressive lesions such as fibrosarcoma. ${ }^{[9]}$

Prominent fascicular or storiform pattern of fibroblasts in the present case was consistant with the cellular variant of BFH. Because of its high recurrence rate regular 1 year follow up was advised to the patient. ${ }^{9}$

The differential diagnosis of oral BFH includes MFH, fibrosarcoma, solitary fibrous tumor, angiomatoid fibroushistiocytoma \& leiomyoma.

Positive immunoreactivity for vimentin and CD 68 demonstrates that the lesion is composed of fibroblastic and histiocyte-like cells. The negativity for S-100 differentiates the lesion from neurogenic tumours, and less than $10 \%$ positivity for Ki-67 demonstrates that the lesion is not aggressive or proliferative. ${ }^{[10]}$

MFH was excluded due to lack of pleomorphic sarcomatous cells, lack of typical and atypical mitotic figures, and lack of bizarre giant cells, necrosis, and prominent areas of haemorrhage. Fibrosarcoma was excluded by the lack of obvious malignant features, invasive margins, and the characteristic herring-bone pattern. SFT was excluded as they pathologically appear as monomorphic spindle cells organised into interlacing fascicles and 'pattern-less' pattern with hemangiopericytoma-like vascular spaces. AFH was excluded as it is characterized predominantly by sheets of histiocytic cells arranged in serpentine pattern having cystic areas of haemorrhage. According to Weiss et al. SMA may occasionally be positive in BFH and their sporadic immunoreactivity should not considered as evidence of smooth muscle neoplasm. Leiomyoma was excluded as they have a more distinct fascicular arrangement with blunt-ended plumper nucleus and cytoplasm showing longitudinal striations corresponding to myofilamentous material. ${ }^{[10]}$

Treatment of choice is usually wide surgical resection along with safe margins of $2 \mathrm{~mm}$ for soft tissue lesions $\& 5 \mathrm{~mm}$ for bony lesions. ${ }^{[4]}$ Regular follow up is must owing to its high recurrence rate. ${ }^{9}$

The present case showed similar microscopic features with positive immunoreactivity for SMA \& CD68 demonstrating the lesion to be composed of fibroblastic \& histiocytic cells. Negativity for S100 ruled out neurogenic tumors \& less than $10 \%$ positivity for Ki67 demonstrated the less aggressive behavior. Complete surgical resection was performed in our case \& one year follow up was asymptomatic.

\section{Conclusion}

Oral BFH parodies many clinical oral lesions \& may often be misdiagnosed initially. It represents a pathologist's phantasm because the histopathological \& morphological patterns are common to many other soft tissue tumors. Thorough clinical examination \& proper diagnostic approach should be followed. Wide surgical resection with safe margins \& regular follow up are recommended.

\section{References}

[1]. Sharon W Weiss, John R Goldblum In Benign Fibrohistiocytic Tumors. Enzinger \& Weiss's soft tissue tumors. Fourth Ed. Atlanta 2001. P. 441-490

[2]. Saluja H, Kasat V, Rudagi B, Dehane V, Kalburge J, Nikam A. Benign fibrous histiocytoma of the maxilla: A case report \& review of literature. Indian Journal of Dental Research 2014; 25(1):115-117

[3]. Shafer's Textbook of Oral Pathology. $7^{\text {th }}$ Ed. 2014. P. 163

[4]. Neville, Damm, Allen, Bouquot in Soft Tissue Tumors. Oral \& Maxillofacial Pathology. $3^{\text {rd }}$ Ed. 2014. P.513-515

[5]. Regezi, Sciubba, Jordan in Connective Tissue Tumors. Oral Pathology: Clinical pathologic correlations $6^{\text {th }}$ Ed. 2013. P. 173

[6]. Jain V, Rathi T, Silva J, Agrawal R, Ullah H. Oral Benign Fibrous Histiocytoma of Chin: A rare Case Report \& Literature Review. IJSS Case Reports \& Reviews 2014; 1(4):8-11

[7]. Pierson J. Dermatofibroma: Background, Pathophysiology \& Epidemiology. Medscape Reference Drugs, Diseases \& Procedures. 2016

[8]. Menditti D, Laino L, Mezzogiorno A, Sava S, Bianchi A, Caruso G et al. Oral benign fibrous histiocytoma: two case reports. Cases Journal 2009; 2(9343):1-5

[9]. Vaidya K, Nagarala T, Sukesh. Cellular variant of benign fibrous histiocytoma: A Case Report. Asian Path. J. Health Sci. 2014; 1(2):502-506

[10]. George A, Pynadath M, Jayapalan C, Manjunath J, Nair R. B. Benign Fibrous Histiocytoma of Maxillary Gingiva. Dentistry ISSN:2161-1122. 2014;4(5):1-3

Figure Legends 


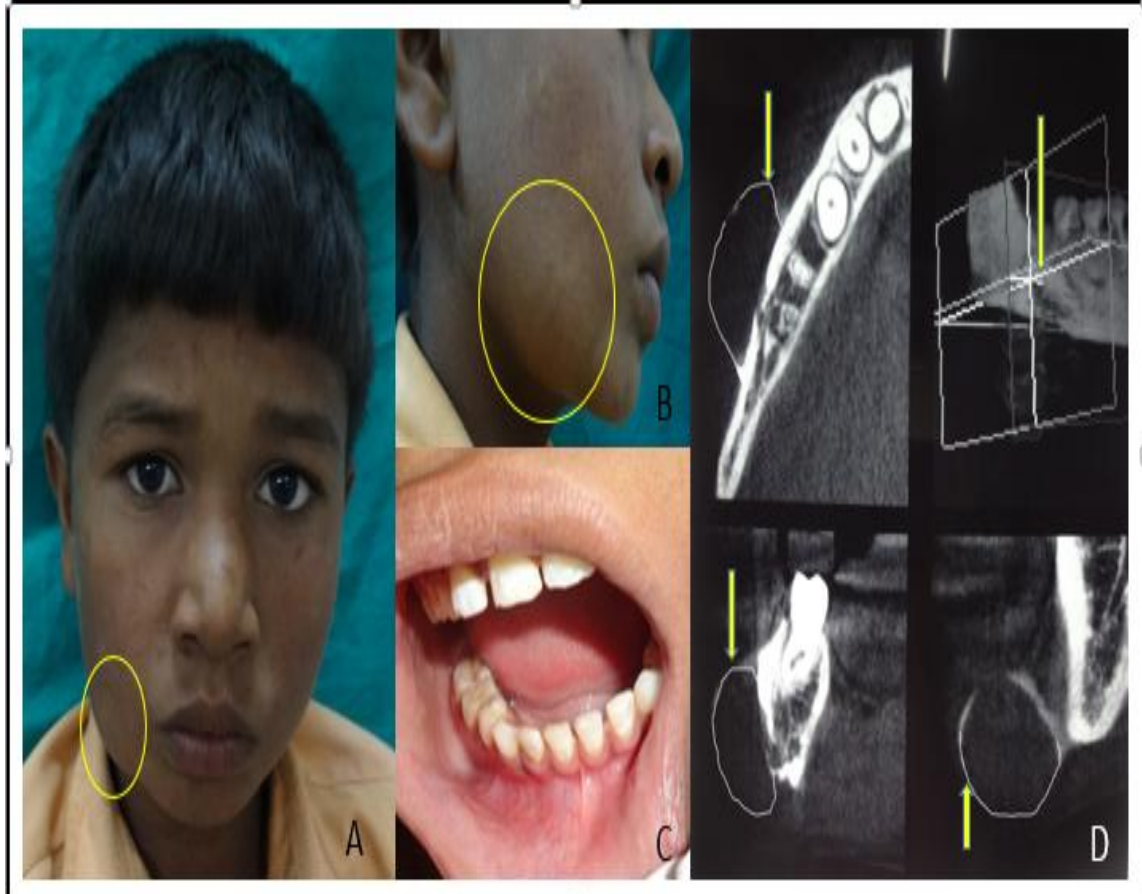

Figure 1: A, B : Extraoral Swelling near the inferior border of Mandible in parasymphysis region; C: Intraorally intact teeth \& oral mucosa; D: CBCT showing well defined paracortical radiolucency in the region of right mandibular D, E \& 6)

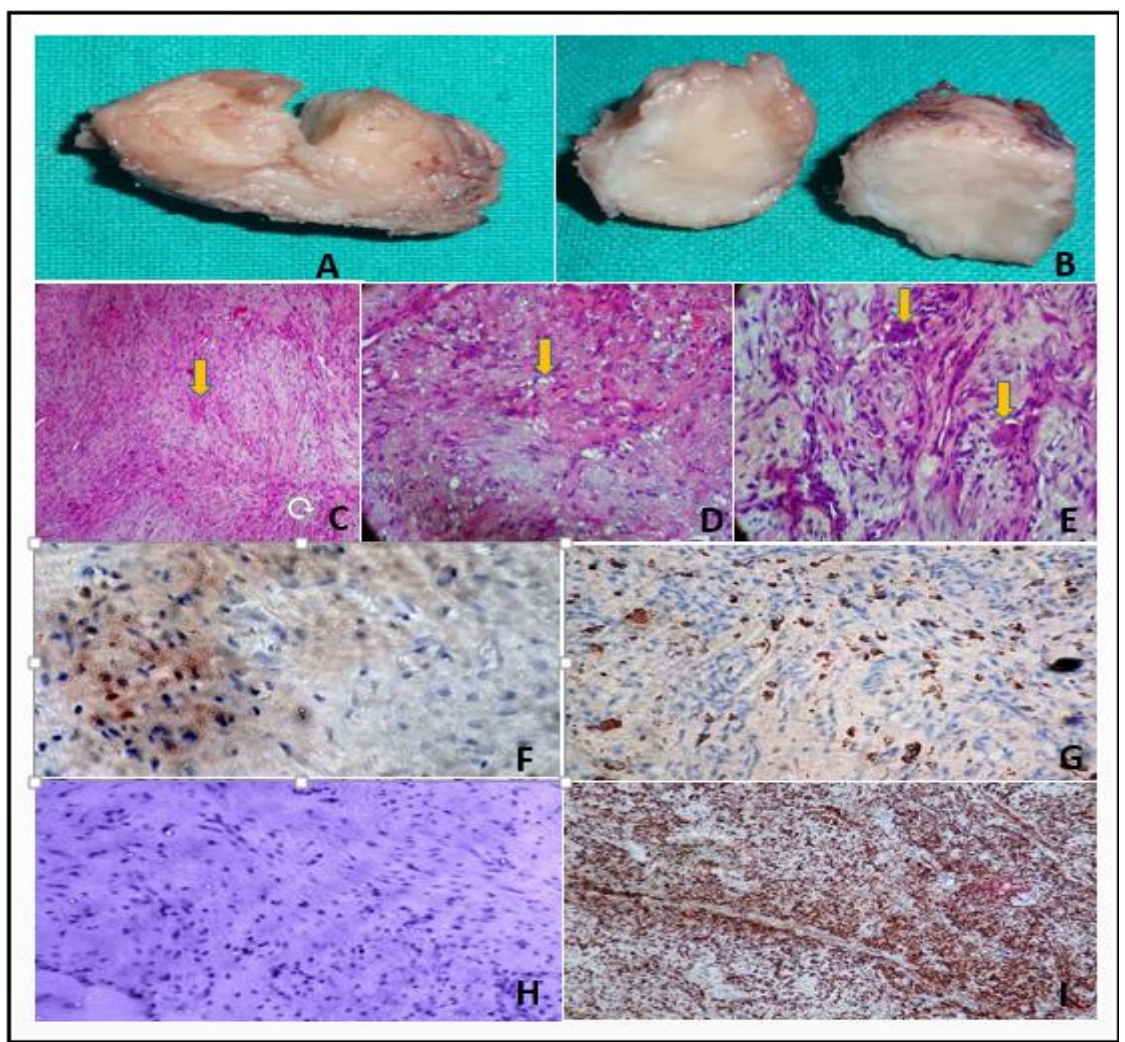

Figure 2: A:Gross Specimen; B: Cut Surface; C, D \& E: Histological images showing fascicular \& storiform arrangement of spindle cells, scattered histiocytes \& touton type giant cells respectively; F, G, H \& I: Immunohistochemical images showing expressions of Ki 67, CD 68, S100 \& SMA respectively. 